\section{Vol. 65, No. RR-1}

In the report, "CDC Guideline for Prescribing Opioids for Chronic Pain - United States, 2016," three errors occurred. On page 1, the last sentence of the Summary should read, "CDC has provided a checklist for prescribing opioids for chronic pain (http://stacks.cdc.gov/view/cdc/38025) as well as a website (http://www.cdc.gov/drugoverdose/prescribing/resources.html) with additional tools to guide clinicians in implementing the recommendations." On page 8, the first sentence of the first full paragraph should read, "NCIPC announced an open meeting of the NCIPC BSC in the Federal Register on January 11, 2016." On page 49, in the fourth line of the Stakeholder Review Group, the affiliation for Gerald "Jerry" F. Joseph should read, "American College of Obstetricians and Gynecologists."

\section{Vol. 65, No. 9}

In the report, "Notes from the Field: Lymphocytic Choriomeningitis Virus Meningoencephalitis from a Household Rodent Infestation - Minnesota, 2015," on page 248 , the first sentence of the fourth paragraph should read, "The family was referred for integrated pest management services through the St. Paul-Ramsey County Department of Public Health, with assistance from the Minnesota Department of Health Healthy Homes grant program." 